\title{
Can rationing of cardiological services be rational?
}

\author{
Douglas Chamberlain, Richard Alderslade
}

In the United Kingdom there is a pervading perception that provision of health resources has not kept up with the availability of effective clinical procedures. By international standards expenditure on health services expressed as a percentage of gross domestic product remains low. ${ }^{1}$ To clinicians the system feels increasingly tight financially because resources are not available to meet expressed clinical demand. This is particularly true of cardiology and cardiac surgery where the National Health Service (NHS) can no longer afford many effective treatments for all who might benefit from them. This harsh fact is often a source of disappointment and frustration. It can also cause conflict between professional groups who struggle to maintain their own service needs against competing interests. Is the problem inevitable? If it is, can we distribute resources in a manner that is seen to be equitable and fair?

\section{Background}

The reasons for the shortfall in provision are manifold, but innovation is the most important-and of special concern in cardiology because the pace of progress has been spectacular. Some cardiologists still active in the discipline were practising when digitalis, quinidine, nitrates, mercurial diuretics, and hexamethonium were the only specific remedies available for heart disease. Surgical options comprised closure of a patent ductus, Blalock anastomoses, and relief of mitral stenosis by digital splitting of fused cusps. Now the available drugs include increasingly specific modulators of myocardial, vascular, and autonomic function, angiotensin converting enzyme inhibitors, antilipidaemic agents, antiarrhythmics, and thrombolytics; all are highly effective but many are very expensive. Interventional cardiology has seen the growth of angioplasty over a decade as well as the slower development of balloon dilatation of valves. Implantable devices have become ever more complex with dual chamber pacing now. commonplace, antitachycardia pacemakers gaining acceptance, and implantable defibrillators poised to play a role in the United Kingdom as they do already in the United States. Cardiothoracic surgeons have been busy for 20 years with coronary grafting but now many are turning to complex antiarrhythmic surgery and transplantation. Many of these developments demonstrably improve prognosis; all are impressive in terms of symptomatic relief. But every new advance increases the dilemma of cardiologists and cardiothoracic surgeons who work to a budget that is set by political and administrative considerations rather than by expressed clinical needs.

Other factors have compounded the economic penalties of innovation. First, important demographic changes are occurring in Britain. The fall of birth rate over the past 25 years together with the growth in the proportion of the elderly has reduced appreciably the percentage of the population who contribute directly to national wealth. The budget for the care of the elderly has grown; new and expensive procedures are increasingly in demand. As age increases beyond 75 years the average medical costs of caring for the elderly increase significantly; the proportion of the population within this bracket has increased by $28.4 \%$ in the past 10 years. $^{23}$ These trends will continue. A second factor adding to the cost of innovation is the increased expectation of a public with a keen appetite for media coverage of medical developments. General practitioners contribute as well, both because of increasing knowledge of recent progress and because one impressive medical or surgical result tends often to trigger several similar referrals. Finally, medical inflation has outstripped general inflation, partly because the health service is labour intensive and salaries have risen in real terms.

The shortfall in cardiological services within the United Kingdom is greater than many perceive. Provision remains grossly uneven and where little is provided expectation remains low. This is no justification for continuing inactivity. We believe that it is inappropriate that in 198845 of 210 health districts in England and Wales did not have a trained cardiologist ${ }^{4}$ or that over 2 million people identified in an earlier survey had no immediate access to temporary pacing for countering a life threatening emergency. ${ }^{5}$

The argument that Western Europe as a whole has more than seven times as many cardiologists proportionally than the United Kingdom and that the United States has 10 times more may not be a strong one. ${ }^{4}$ But it may be relevant to ask whether financial constraints were, historically, the only reason for this disparity? Perhaps they were not. In the United Kingdom we have currently a system that is neither directly responsive to market forces nor subject to strict central control. Priorities are determined at a local level by a process that is administrative and locally "political", often without a full appreciation of the weight of competing needs.

Even such provision as is available may not 
be used efficiently. In strictly economic terms it makes little sense to equip a catheter laboratory at a cost of over $£ 500000$, yet see it inadequately staffed by radiographers, technicians, and nurses so that plant lies idle for many daytime hours: a situation familiar enough in many cardiac centres. Within district general hospitals the implications of such poor support are less dramatic but equally disruptive of the service. Almost always levels of technical staff are below reasonable recommendations; occasionally these staff are not provided at all. Simple but essential equipment such as treadmills often remains idle because physiological measurement technicans are scarce. So too is secretarial help.

Such critical limitation of resources has another serious implication: the expensive resource of cardiological skill may be inhibited or misdirected to inappropriate tasks. Undue priority may be given to some tasks at the expense of others that may be as essential but less urgent. Within catheter laboratories emergency procedures are displacing urgent cases from the waiting list; the pressure is compounded by the need to introduce angioplasty without extra resources being available. In the district general hospital crisis management displaces any resolve to introduce service improvements; for example, very few hospitals have rehabilitation programmes or adequate resuscitation training although the need for such facilities is not contentious and the cost modest.

At the academic level within cardiac centres and academic departments a major priority has become the preservation of research capability: thus studies that are economically safe-notably those financed by pharmaceutical companies-can take precedence over the pursuit of more fundamental research. Risk is an invariable component of true innovative research work, but risk is a luxury that few research directors can now afford.

\section{Solutions}

Is more money likely to be forthcoming? How satisfied are we that the money we currently have available is always being used wisely? How rational is its distribution? How will future changes (for example the implementation of the white paper Working for Patients ${ }^{6}$ ) affect this situation of perceived scarcity? How should cardiologists respond now and in the future to their current difficulties?

Before attempting to answer these questions, two processes need to be understood: allocation and rationing. Their edges are often blurred. But it is important to consider them separately, with allocation as the key issue. Allocation within a publicly funded system such as the NHS is a political and administrative process. The government decides how much it will commit to the NHS against other competing demands and the Department of Health, the regional health authorities, and district health authorities allocate resources in an attempt to provide a volume of resource across their entire range of statutory responsibilities.

This process of allocation is often confused with rationing. Rationing accepts a given allocated volume of resource and attempts to achieve a defined objective: the maximum beneficial health effect within a given scale of provision. Within the NHS rationing has always been a clinically based activity. Decisions on priority for admission and treatment are made by clinicians according to clinically determined criteria. This responsibility is the other side of the coin of clinical freedom. The stringency of rationing depends on the adequacy of allocated resources and fairly modest increases in allocation, particularly if sustained, might remove many of clinicians' current problems.

How rational can we make allocation? The truth is that since the 1950s health has been relatively well treated compared with other major publicly funded sectors, ${ }^{1}$ and it is probably unreasonable to expect that public funding of the NHS will increase in real terms by more than $1-2 \%$ a year, despite a widely shared perception of need. Total expenditure on health could be increased by a greater reliance on the private sector and charitable and voluntary resources. There is also the drive within NHS management towards greater efficiency and productivity in the use of available resources.

The decisions taken by regional and district health authorities on the allocation of funding to cardiological and cardiac surgical services are influenced by three mechanisms.

\section{POLITICO-VOCAL MECHANISM}

The politico-vocal mechanism-"who shouts loudest gets most"- has a long and distinguished tradition. It encompassess the use of "political" pressure groups, including public sympathy and emotion. In a service where the objectives are set by political considerations and considerations of personal value, there may be no alternative to being guided by the process described as "disjointed incrementalism".?

\section{RATIONAL-DISTRIBUTIVE MECHANISM}

This originates with the perception that neither the providers nor the consumers of health care are competent to assess national need. The conventional response is to require epidemiologists and health system managers to identify these total population needs and establish programmes of service activity designed to secure health improvements. Some planners and managers believe that the introduction of any new technology should be supported by evidence from randomised controlled trials, but they may not always understand the limitations of such trials. Trials offer the best guidance we have for the efficacy of treatment but there is often room for a different interpretation and rejection of what may seem definitive conclusions. Moreover, trial results are never wholly relevant to an individual patient with his or her own unique set of pathological and functional problems.

Despite these uncertainties, planners and 
managers also increasingly expect additional evaluative information, which can be equally difficult to provide and interpret. They want to know not only that a procedure is effective but also how effective and at what cost. Cost utility analysis is one method designed to consider the cost of new technologies against their potential global population benefit. Mortality benefit is adjusted for the quality of life during the life years gained by the application of the technology. This measuring tool is termed the QALY-Quality Adjusted Life Year. There are various difficulties associated with this approach and it has received substantial criticism.

While the basic principle of cost-utility analysis cannot be challenged its implementation is more difficult than many believe. What is cost and how is it measured? Should judgments be made based on cost to the hospital or to the NHS? The two can be very different. Should we be concerned with marginal costs or full costs? Should costs be calculated per procedure or per case? Should the calculation be for this admission, this year, or forever? Even if the guidelines are agreed, measurement is complex, as wide disparities in data (for example the cost of coronary artery bypass grafting) can attest. How easy is it to estimate outcome? Many feel it is not easy, and that attempts to do so may lead to misleading distortions. Although the QALY is likely to become established as a permanent package within health economics, it is at best a crude measure and may be based on questionable assumptions.

Nevertheless, despite these limitations, the technique can be useful in guiding decisions on allocation and in extending the framework of "rationality" on which they are based. For example the results of ISIS-2 show the clinical effectiveness of treatment with streptokinase as well as aspirin after infarction. ${ }^{8}$ It can be shown by cost-utility analysis ${ }^{9}$ that the costs per QALY of adding streptokinase into the treatment required are low compared with those costs published for other procedures in routine use, including mammography, heart transplantation, coronary artery bypass grafting, and all types of treatment for end stage renal failure. ${ }^{1011}$

\section{MARKET ORIENTATION}

A pure market orientation is inappropriate within health care provision because those most in need are often least able to pay. For this reason the proposals within the NHS white paper aim to continue to base funding largely upon taxation, with receipt of service remaining substantially free at the point of delivery. Publicly funded authorities will determine the health care needs of the population and will purchase services to meet those needs from a range of service providers, within both the public and private sectors. Increasingly these "purchasing" authorities will not manage services directly. "Providers" will deliver service against contracts established with the "purchasing" authorities; the competitive forces created within this marketplace are expected to create increased efficiency and eliminate the inefficiencies inherent in any large bureaucracy. The authors of the white paper believe that this greater efficiency will be associated with a better quality of care and that standards can be set and monitored within the contracts. A substantial investment will be necessary in information systems to support the contractual interface between purchasers and providers. Will the expected benefits be achieved? The answers to this question lie in the future, though the challenge is immediate.

\section{The difficult way forward}

It is clear that cardiologists and cardiac surgeons must be involved in decisions about both allocation and rationing if they are to influence these processes for the benefit of patients. Yet this object poses an inevitable dilemma. Should doctors face the costs of their decisions? If they do not, the result can be inefficiency and favoured provision of patient care for the few to the detriment of the many. If they do, then the result could theoretically be a downward pressure on the quality of service.

We have no easy solution. Most agree that the clinical views should be heard at the level of political allocation, at the level of local administrative distribution of the allocation using the most appropriate "rational" means, and within the unit itself, increasingly through an involvement in resource management. This involvement in resource management may reduce anomalies in the provision of health care but has little effect on the adequacy of allocation.

Clinicians must accept that if treatment of proven effectiveness is not available for all who may benefit, an element of rationing must be introduced if provision is to be better than random; but this poses considerable problems when dealing with individual patients. Physicians and surgeons are already familiar with triage as the technique invented by military surgeons for selecting battlefield casualties most likely to benefit from the application of scarce resources. But when this process has an economic basis it is less familiar and less welcome. It creates a even greater dilemma because it influences the doctorpatient relationship. The patient regards the physician as a trusted adviser, and expects him to be, where necessary, his advocate.

How can physicians combine their clinical roles with their responsibility for the use of resources? No one but the physician can make rationing decisions based on clinical criteria. Yet their actions must not damage their relationship with an individual patient. Again we have no ready answer, but only a part solution. Physicians will come to see that involvement in the processes of medical audit and resource management can lead increasingly to informed decisions based upon knowledge of outcomes that are translated into agreed protocols for service delivery. In this way doctors should not need to introduce budgetary considerations into clinical decisions about individual patients. They will recognise the position of their patient in relation to a 
predetermined guideline or protocol for availability of treatment. It seems acceptable that they should act accordingly, but ideally with the full understanding of the patient. In this way physicians can - at least to a degreebe accountable to their patients; to the organisation in which they work, to its resources; and to society in general.

Unfortunately many observers of the medical scene, including some managers and health economists, may have an overly simplistic view of the dilemma. Decisions on clinical matters can never be made according to rules that are universally applicable, especially when their basis is clinical trials that always have important limitations. Other approaches have been recommended, but are equally fallible. We have heard strident calls for mortality data to be made public as a guide to the "consumer". Is this fair to the surgeon who is prepared to offer hope to a high risk patient? Even more importantly is it fair to patients in that category? Similar problems would apply were morbidity data made public as a guide to the "consumer".

\section{Conclusion}

We conclude that there is a continuing need for judgment in the application of trial data and measures of cost-utility and a need to use the best available basis for treatment decisions in the absence of such data. Fortunately these conclusions can reasonably be reconciled with the concept of clinical freedom.

Many believe that the obituary for clinical freedom was premature. ${ }^{12}$ Perhaps the meaning of freedom has been misunderstood. It does not imply licence. We are free to drive where we will. But not to drive on the wrong side of the road, or too fast, or against traffic lights. Likewise, clinical freedom means the right to make decisions that do not flout a wider interest. Clinical freedom-in this correct and valid sense-must be respected and defended.
Those who exercise this freedom will welcome a greater involvement in audit and peer review and in resource management, which-applied with wisdom-must be adopted to provide information within the peer group on criteria for sensible clinical rationing.

The gap between what is technologically possible and what can be funded publicly will grow. Allocation can never be totally rational, and the NHS will continue to operate within a political context. Many expectations will not be met. Clinical rationing will require more attention to audit and to the creation of agreed treatment protocols, based on evaluation. If cardiologists and cardiac surgeons do not accept their rationing role in clinical management others will.

This paper is based on presentations at a Cardiovascular Workshop in June 1989 arranged by Mr Dimitri de Grunwald for Bayer Pharmaceuticals whose help and encouragement is gratefully acknowledged.

1 Teeling Smith G. Health: the politician's dilemma, London: Office of Health Economics, 1986.

2 Office of Population, Censuses and Surveys. Population estimates 1979. PP1. No 4. London: Her Majesty's estimates 1979. PP1.

3 Office of Population, Censuses and Surveys. Population projections. PP2. No 16. London: Her Majesty's Stationery Office, 1989

4 Chamberlain D, Bailey L, Sowton E, Ballantyne D, Boyle D McC, Oliver M. Staffing in cardiology in the United Kingdom 1988. Fifth biennial survey. Br Heart $J$ 1989;62:482-7.

5 Chamberlain D, Bailey L, Julian D. Staffing and facilities in cardiology in the United Kingdom 1984. Third biennial survey. Br Heart $J$ 1986;55:311-20.

6 Department of Health. Working for patients. London: Her Majesty's Stationery Office, 1989.

Braybrook D, Lindblom C. A strategy for decision. New York: Free Press, 1963.

8 ISIS-2 (Second International Study of Infarct Survival) Collaborative Group. Randomised trial of intravenous streptokinase, oral aspirin, both, or neither among 17187 streptokinase, oral aspirin, both, or neither among 17187
cases of suspected acute myocardial infarction: ISIS-2. cases of suspected acute

9 Trent Regional Drug Information Service. Therapeutic forum. No 2 Trent Regional Health Authority, January 1989.

10 Department of Health. Breast cancer screening: the Fores report. London: Her Majesty's Stationery Office, 1986.

11 Williams $A$. The economics of coronary artery by-pass grafting. Br Med J 1985;291:326-9.

12 Hampton JR. The end of clinical freedom. $\mathrm{Br}$ Med $J$ 